\title{
Foreword
}

\section{Echocardiography 1996}

In this third consecutive issue of the British Society of Echocardiography supplement on echocardiography we continue the theme of multi-author invited review articles on practical topics related to clinical practice. The daily practice of echocardiography may differ from one institution to another. Therefore, we encourage articles that involve more than one institution and come from different countries so that the views expressed are a consensus. Indeed, this democratic attitude forms the basis of the British Society of Echocardiography and helps promote a practical approach to echocardiography.

Using this philosophy a consensus has emerged from several top European echocardiographic centres on quantifying aortic and mitral valve stenosis. What becomes immediately apparent is that surgeons operate on patients not video tapes or gradients and pressure half times. Therefore the final decision on the patient's management must remain with the clinician who should be appropriately informed by the echocardiographer. The authors briefly review some important basic concepts on Doppler and describe the relative merit of cross sectional imaging and Doppler, pointing out some of their limitations. They also discuss some of the problems related to Doppler measurements of prosthetic valves.

The topical subject of quantification of ventricular systolic function is elegantly discussed by Nelson Schiller. This is a controversial subject, particularly as there is a tendency for the pharmaceutical industry to drive clinical practice based on some multicentre trials. The obvious danger of this is that one number, which may or may not represent left ventricular function, can be derived by several methods with varying degrees of reliability. Schiller correctly stresses the importance of individual expertise and reproducibility of the laboratory in which the measurements have been made. Here there is a clear need for "accredited" echocardiographers to perform and interpret measurements because the therapeutic implications for the patient may be grave.

Diastolic function is another important and topical subject. Abnormalities are often responsible for patients' symptoms. Diastolic function is difficult to assess objectively and it is even more difficult to measure. Traditionally, radionuclide techniques have been used to measure diastolic function. However, the introduction of Doppler echocardiography has revolutionised diastology. In this issue, Yamamoto and Nishimura review in detail the principles behind Doppler assessment of diastole and carefully emphasise the multitude of conditions that may alter the various parameters. It is an important article clarifying, in the most elegant way, the various pathophysiological mechanisms involved in diastolic function.
Two articles by McDicken on Doppler technology are based on a one day workshop on echo technology organised by the British Society of Echocardiography. In these articles, up to date and detailed reviews of modern technology are provided by experts. These reviews will prove to be important for physicists and echocardiographers as well as a useful adjunct for clinicians who may lack the understanding of basic ultrasound technology. Of particular importance is the description of the principles of Doppler tissue imaging, myocardial backscatter, and myocardial contrast echocardiography. Myocardial contrast echocardiography may well provide vital perfusion information to complement traditional echocardiography data in the future.

Sixteen abstracts were presented to the annual British Society of Echocardiography meeting held October 1995 in Coventry. These are published in this supplement. As expected, both the submission and subsequent rejection rate of abstracts has increased year on year. However, the total number of presented abstracts was slightly reduced to facilitate increased discussion. We now have plans to add poster presentations to accommodate growing academic interest in this meeting.

The British Society of Echocardiography is well established as the official representative body of the British Cardiac Society on echocardiographic matters. In 1996 we had more than 1000 members, $60 \%$ being nonmedical staff. This clearly reflects echocardiographic practice in the United Kingdom. It is our intention not only to continue promoting the technique in this country but also to integrate practice with that of the rest of Europe. To this end, we have increased our contact with the European Working Group on Echocardiography. Since the practice of echocardiography differs between the various European countries it is our aim to continue improving training and accreditation of individuals rather than to dictate unrealistic practice guidelines.

May we take this opportunity to remind you that October 25-26 is our next meeting. If you wish to obtain abstract forms please write to the British Society of Echocardiography.

Finally, we wish to express our gratitude to Nycomed Imaging for sponsoring this year's supplement on echocardiography.

M J MONAGHAN

Past President $\mathcal{E}$ Council Member P NIHOYANNOPOULOS Chairman

Research and Scientific Subcommittee, British Society of Echocardiography, 9 Fitzroy Square, London W1P $5 A H$ 